\title{
Immunoreactivity of adsorbed anti human chorionic gonadotropin studied with an optical waveguide interferometric sensor
}

\author{
Rene G. Heideman, Rob P. H. Kooyman," Jan Greve \\ University of Twente, Department of Applied Physics, Applied Optics Group, MESA Research Institute, \\ P.O. Box 217, 7500 AE Enschede, The Netherlands. \\ Tel: [31] 53 3067. Fax: [31] 53309549
}

(Received 11 August 1992; revised paper received 7 January 1993; accepted 10 February 1993)

\begin{abstract}
A study on the immunoreactivity of adsorbed $\alpha$ hCG molecules as a function of substrate hydrophobicity and protein coverage is presented. The experiments were performed with a planar waveguide interferometrical immunosensor. The substrate hydrophobicity and the antibody density were found to be of major importance for the immunoreactivity of the adsorbed antibodies. A combination of a hydrophobic (contact angle $\geq 80^{\circ}$ ) substrate and only a fraction $(\approx 0.3)$ of a monolayer of antibodies gives the highest immunoreactivity. However, the immunoreaction affinity constant of the active antibodies is found to be essentially independent of the substrate hydrophobicity. The experimental results indicate that the orientation and not the conformation of the active antibodies on the substrate is dependent on the substrate hydrophobicity.
\end{abstract}

Keywords: immunosensor, immunoreactivity, adsorbed antibodies, interferometer, planar waveguides, hydrophobicity, coverage.

\section{INTRODUCTION}

The development of direct optical immunosensors has been a challenging topic in the last decade (Place et al., 1985; Robinson, 1991). These immunosensors do not require labelling of antigen molecules and offer the opportunity of studying reaction kinetics. The Surface Plasmon Resonance (SPR) sensor (Jönsson et al., 1991; Karlsson et al., 1991) is the most developed type in this

* To whom all correspondence should be addressed. field. The grating coupler concept gives rise to a new class of sensors (Spohn \& Prenosil, 1990). The SPR and the grating coupler sensors show similar sensitivities (Barner et al., 1991; Lukosz, 1991). A new development concerns the use of interferometrical immunosensors based on planar waveguides. Examples are the difference interferometer (Fattinger et al., 1992) and the MachZehnder interferometer sensor (Heideman et al., $1991^{2}$ ) denoted as the PIWI (an abbreviation for the words planar interferometric waveguide immunosensor). The intrinsic sensitivity of this class of evanescent wave sensors is linear with 
the interaction length used. In practice an approximately ten times higher sensitivity is obtained as compared with the SPR and the grating coupler sensors (Heideman et al., 1992').

The sensing principle of the PIWI is the interferometric detection of the changing optical configuration of a (reusable) planar optical waveguide. Only changes in the refractive index profile occuring within the evanescent field region of the mode travelling through the waveguide will cause such a change. Evanescent field sensors are in principle sensitive to both biochemical surface reactions and bulk refractive index changes. The high sensitivity of the PIWI arises from an optimized waveguide structure and a relatively long interaction length $(1 \mathrm{~cm})$ in combination with a Mach-Zehnder interferometer detection scheme. The latter offers the opportunity of an easily accessible reference arm allowing for compensation for common mode effects such as temperature variations. When biochemical surface reactions are studied, the reference arm of the PIWI also compensates for bulk refractive index changes.

A successful immunosensor is not only a device with a high intrinsic sensitivity; the activity of the immobilized receptor layer is an equally important factor (Matson and Little, 1988; Jönsson et al., 1991). In order to obtain an optimized receptor layer coating procedure, it is important to study the immunoreactivity of adsorbed antibodies. From previous studies it is known that the nature of the adsorption process of antibodies is strongly dependent upon the protein concentration and the hydrophobicity of the substrate (Norde, 1986; van Damme, 1990), suggesting a dependence of immunoreactivity on substrate hydrophobicity. Also the antibody density on the substrate is known to be important for the immunoreactivity (Matson, 1988). With the PIWI these suggestions were investigated, using the waveguide surface as the substrate for an adsorbing (monoclonal) antibody. The hydrophobicity of the waveguide surface as well as the coverage fraction of adsorbed antibody were varied. A series of immunoreactions with increasing antigen concentration were measured. The results obtained provide some insight into the behaviour of antibodies at substrates, and can be used to improve the receptor layer coating procedure.

\section{MATERIALS AND METHODS}

\section{Reagents}

The IgG protein anti human chorionic gonadotropin ( $\alpha \mathrm{hCG}, \mathrm{MW} \approx 150 \mathrm{kD}$ ) clone 147B and human chorionic gonadotropin (hCG, MW $\approx 40 \mathrm{kD}$ ) were provided by Organon International (Oss, The Netherlands). Anti human serum albumin ( $\alpha$ hSA, $M W \approx 150 \mathrm{kD}$ ), human serum albumin (hSA, $M W \approx 65 \mathrm{kD}$ ) and bovine serum albumin (bSA, $M W \approx 65 \mathrm{kD}$ ) were bought from Sigma Chemical Co. (St. Louis, MO, USA). All other chemicals used were of analytical grade.

The protein solutions were made using a phosphate buffered saline (PBS) solution with $\mathrm{ph}=7.35$ and osmolality of $290 \mathrm{mOsmol} / \mathrm{kg} \mathrm{H} \mathrm{H}_{2} \mathrm{O}$. The refractive index of the PBS solution is $1.3376 \pm 0.0002$, as measured with an Abberefractometer. The refractive index of the pure water used is 1.3358 . These values were determined at $\mathrm{T}=296 \mathrm{~K}$ and $\lambda=633 \mathrm{~nm}$. It should be noted that in view of the small difference of this He-Ne-laser wavelength and those of the Dlines of sodium $(589 \mathrm{~nm})$, at which wavelength the reading of the Abbe-refractometer is calibrated, the above mentioned refractive indices might be slightly off the actual values. However, in the following only the difference between the two quoted refractive indices will be relevant, and consequently, the absolute offset is immaterial. The refractive index of the proteins $\alpha \mathrm{hCG}$ and hCG is assumed to be 1.45 (Cuypers et al., 1983).

\section{Apparatus and waveguide structure}

The PIWI consists of a Mach-Zehnder interferometric set-up (see Fig. 1), combined with an optimized planar waveguide structure as the sensing element (see Fig. 2). The planar waveguide is easily cleanable without damage using standard techniques including soaking in strong acids. The details of this sensor have been reported elsewhere (Heideman et al., 19912; Heideman et al., 19921,2). The signal obtained from this sensor is an optical phase change $\Delta \Phi$, which is a measure of the change in the refractive index profile at the waveguide surface. The minimum phase change that can be detected is $\Delta \Phi \cong 0.01 \cdot 2 \pi$ per hour (see also section Immunoreaction experiments). 


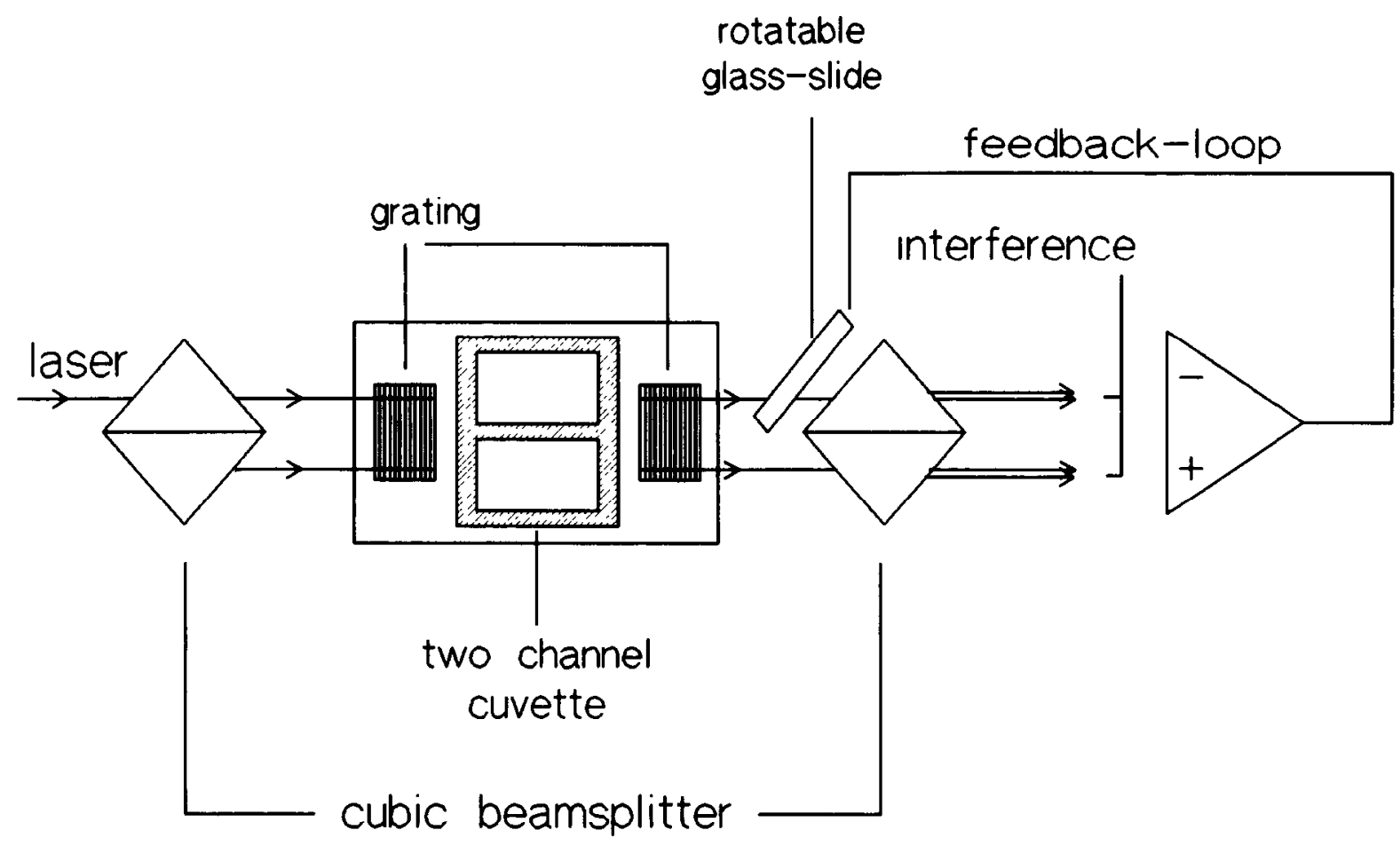

Fig. 1. Mach-Zehnder interferometrical set-up. The first and the second cubic beamsplitters are used to split respectively to recombine the laserbeam(s). Two interference patterns are obtained. The difference between the two measured intensities is regulated to zero using a rotatable glass-slide (which provides a variable phase shift) and a feedback-loop. The regulation current is used as the signal.

\section{Characterization of the waveguide surface}

The hydrophobicity of the waveguide surface is characterized by measuring its contact angle (Moore, 1972). We used a home-made instrument to measure the contact angle at room temperature of a droplet of pure water of $\approx 5 \mu l$ on the waveguide surface. The contact angle measurement should take place right after the deposition of the droplet in order to avoid evaporation artefacts. The resolution of the apparatus is $\pm 2^{\circ}$. The values of the contact angles used in this paper were measured directly prior to recording of the adsorption experiments. The surface roughness of the waveguide was found to be of negligible influence on the contact angle value: this was checked with contact angle measurements on the polished and the non-polished side of a Si-wafer. For both a thermally oxidized $(\theta=$ $\left.10 \pm 2^{\circ}\right)$ and a PTFE coated wafer $\left(\theta=98 \pm 2^{\circ}\right)$, these values were the same within the measurement accuracy, while in both situations the surface roughness magnitudes on the polished and non-polished sides were in the order of $\mathrm{nm}$ and $\mu \mathrm{m}$, respectively.

\section{Preparation of the waveguide surface}

Before the measurements, the waveguide is thoroughly cleaned. This consists of soaking at room temperature $(295 \mathrm{~K})$ in neat $\mathrm{HNO}_{3}$ for 10 minutes, thoroughly rinsing with pure water (demineralized, deionized), soaking in $70 \%$ $\mathrm{HNO}_{3}$ at $365 \mathrm{~K}$ for 15 minutes, and again thoroughly rinsing with pure water. Immediately after this cleaning procedure the contact angle of the $\mathrm{Si}_{3} \mathrm{~N}_{4}$ is $20^{\circ}$. When stored in air this value increases to $\approx 40^{\circ}$ within 24 hours. A hydrophobic waveguide surface can be obtained by treating the cleaned structure with Hexamethyldisilazane (HMDS). This treatment consists of steaming the waveguide with pure water vapour $(373 \mathrm{~K})$ and HMDS (Merck) vapour $(373 \mathrm{~K})$ for one hour, respectively, followed by rinsing with acetone. The water vapour provides the waveguide with excess $\mathrm{OH}$-groups, necessary 


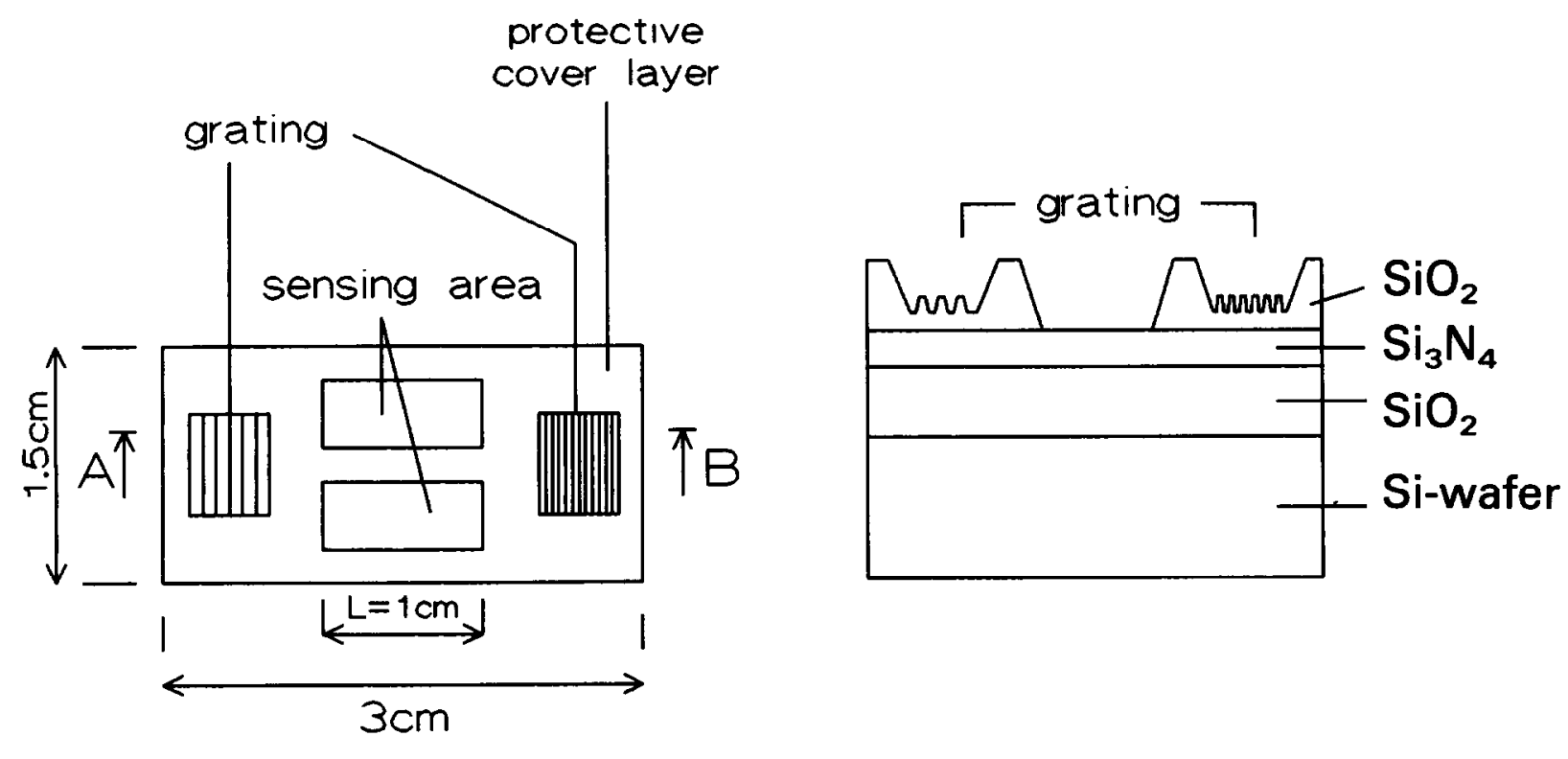

a

b

Fig. 2. The planar waveguide structure used. (a) Top view. (b) Cross-section of the waveguide through the line A$B$. The gratings (with grating constants $4 \mu \mathrm{m}$ and $2 \mu \mathrm{m}$ resp.) are used to couple light in and out of the waveguiding $\mathrm{Si}_{3} \mathrm{~N}_{4}$-layer, which has a thickness of $\cong 95 \mathrm{~nm}$ and a refractive index of 2.00. Between the gratings and the sensing areas a cuvette can be pressed to the waveguide. Here $a \approx 1.5 \mu \mathrm{m}$ thick $\mathrm{SiO}_{2}$-layer provides optical isolation from the $\mathrm{Si}_{3} \mathrm{~N}_{4}$-layer.

for covalent coupling of HMDS. The contact anglc is $88^{\circ}$ after this treatment. Storage in air does not significantly affect the hydrophobicity of this HMDS surface: after a week in storage the same contact angle is measured. Intermediate values of hydrophobicity of the waveguide surface were obtained by slightly etching the structure with an HF-solution after the cleaning procedure. The HMDS treatment on such a slightly etched waveguide resulted in a contact angle of $60^{\circ}$. Repeated standard cleaning gradually increases the contact angle to $88^{\circ}$ again. The etching procedure consists of dipping the cleaned structure in an HF: $\mathrm{NH}_{4} \mathrm{~F}$ (1:7) (Merck) solution for a few seconds, followed by thoroughly rinsing with pure water. Because of the low etch rate of the waveguiding $\mathrm{Si}_{3} \mathrm{~N}_{4}$ layer in this $\mathrm{HF}$-solution ( $\cong 0.7 \mathrm{~nm}$ per minute) its thickness and therefore the sensor sensitivity is hardly decreased.

\section{Preparation of the sensor and sensitivity check}

The waveguide structure is placed in the MachZehnder interferometer set-up (see Fig. 1). Between the two grating couplers a two channel
PTFE cuvette is mounted. The dimensions of the channels are $13 \mathrm{~mm}$ length $\times 5.5 \mathrm{~mm}$ width $\times 14 \mathrm{~mm}$ height. It is pressed to the protective $\mathrm{SiO}_{2}$ layer (see Fig. 2) of the waveguide with a silicon rubber seal in between. Then the two channels are filled with $0.8 \mathrm{ml}$ pure water. The measured phase change caused by the subsequent replacement of water by PBS in both the measurement and reference channel is used as a sensitivity check. The bulk refractive index difference is $(1 \cdot 8 \pm 0 \cdot 2) \cdot 10^{-3}$. The corresponding measured phase change is $(4 \cdot 5 \pm 0 \cdot 3) \cdot 2 \pi$, which is in good agreement with theory (Heideman et al., 1992 ${ }^{1}$ ). It should be mentioned in this context that the PIWI is a difference sensor. Effects occuring in both channels at the same time are not monitored.

\section{Adsorption experiments}

After the sensitivity checks the measurement channel is filled with a protein containing PBS solution. This is done by a substitution of half of the channel contents using a pipette. Thereafter, the channel content is homogenized. The resulting adsorption process is monitored. 
Although the adsorption kinetics could easily be followed, we will focus in this communication only on the equilibrium data; the experimental conditions were not such that a meaningful interpretation of the kinetic data is possible, as would be the case when using a well dimensioned flow-through system.

\section{Immunoreaction experiments}

\section{Coating of the receptor layer}

In all immunoreaction experiments to be reported, the general procedure used is the same. The two channels are always treated successively so that effects due to adding, mixing, diluting etc. can be monitored directly. As a first step in the coating procedure the measurement channel is filled with an $\alpha$ hCG-containing PBS solution. After reaching the desired degree of adsorption, the channel is washed thoroughly by repeated flushing with PBS. Subsequently, the reference channel is treated similarly. The next step in the coating procedure consists of filling the measurement channel with PBS containing a high concentration of bSA $(5 \mathrm{mg} / \mathrm{ml})$. This blocking step prevents non-specific adsorption (Hardy, 1986). After 15 minutes, this is followed by thoroughly rinsing using repeated flushing with PBS. Hereafter the same blocking procedure is applied to the reference channel.

\section{Immunosensing}

After coating the two channels we wait until the drift is $\approx 1 \cdot 10^{-2} \cdot 2 \pi$ per hour, before the immunoreaction experiments are started. This value is obtained after a stabilization period of approximately one hour. This differential drift occurs only after the coating step described in the previous section. Apparently in the two coated channels that have inevitably slightly different optical pathlengths, a small fraction of the adsorbed antibodies desorbs; that it is only a small fraction can be concluded from the absence of any non-specific interaction, as discussed below. Although corrections can be made for this drift we regard that $1 \cdot 10^{-2} \cdot 2 \pi$ as the minimal phase change which can at present be unambiguously detected in immuno experiments as described below.

In the immuno experiments half of the cuvette content of the measuring channel is replaced by an hCG-containing PBS solution and half of the cuvette content of the reference channel by PBS.
The solutions are not stirred. Once equilibrium is reached the procedure is repeated with an increased concentration of hCG, until a saturation level is reached. This level corresponds to complete antigen coverage of all available active binding sites. After application of a concentration of $1 \cdot 10^{-9}$ Molar hCG (far from saturation) a 20 fold excess of hSA is added to check the specificity of the reaction. In none of the experiments was a positive response to this hSA concentration observed (Heideman et al., 1992 ${ }^{1,2}$ ), which clearly demonstrates the specificity of the observed immunoreactions.

Finally, the whole immunoreaction procedure is repeated in the reference channel.

\section{RESULTS}

\section{Adsorption of ahCG, ahSA and hSA to a hydrophobic/hydrophilic surface}

As a first step in the immunoreactivity measurements, the antibody $\alpha$ hCG was adsorbed to the waveguide surface. The maximum obtainable $\alpha$ CG concentration was limited to $\cong 4 \cdot 10^{-7} \mathrm{M}$. With the similar IgG antibody $\alpha \mathrm{hSA}$ and with the much smaller antigen hSA higher concentrations could be obtained. In Figs. $3 a$ and $3 b$ the equilibrium phase changes are plotted as a function of concentration, for two extreme values of the hydrophobicity (contact angles $20^{\circ}$ and $88^{\circ}$ ).

From this figure it follows that the affinity of the protein to a hydrophobic substrate appears to be somewhat larger, in line with earlier observations (Norde, 1986; MacRitchie, 1972); however, the maximum equilibrium phase changes seem to be mostly dependent upon the protein used rather than on the substrate hydrophobicity. Saturation of the adsorption process for $\alpha \mathrm{hCG}$ and $\alpha \mathrm{hSA}$ results in a maximum phase change of $\cong 35 \cdot 2 \pi$. It can be calculated (Heideman et al., 1992 ${ }^{1}$ ) that this value corresponds to a homogeneous protein layer with a thickness of $\cong 6 \mathrm{~nm}$ (see appendix, equation (A4)) assuming a refractive index of 1.45 (Cuypers et al., 1983). This layer thickness is in agreement with the expected monolayer value (Valentine \& Green, 1967). Therefore (35 \pm 2$) \cdot 2 \pi$ will be taken as the phase change corresponding to an $\alpha \mathrm{hCG}$ monolayer. 


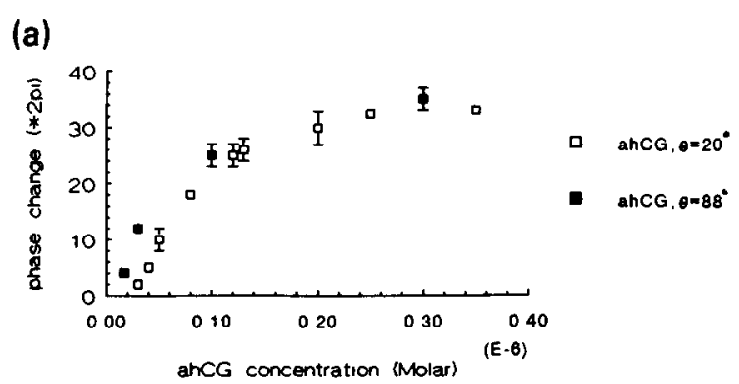

(b)

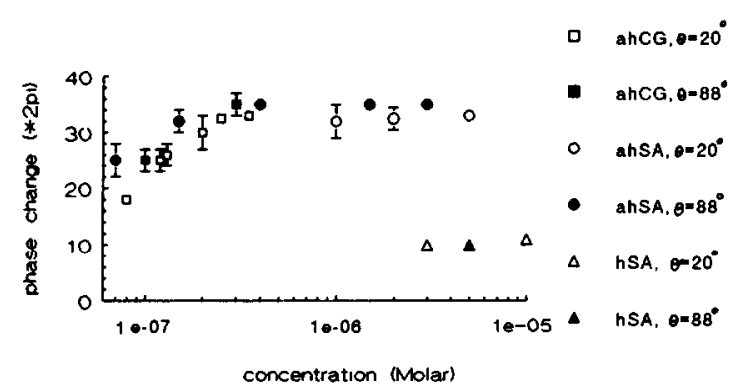

Fig. 3. Adsorption experiments on hydrophilic (hollow marker) and hydrophobic (filled marker) substrates. (a) Equilibrium phase change as a function of ahCG concentration. (b) Equilibrium phase change as a function of protein concentration. Error bars not shown are covered by the marker.

\section{Immunoreactivity with varying $\alpha$ CG density as} a function of substrate hydrophobicity

In this section the coverage with adsorbed $\alpha \mathrm{hCG}$ is varied and the corresponding immunoreactivity is measured. The phase change corresponding to the saturated immunoreaction is taken as the immunoreactivity value. The measurements were done with both the hydrophilic (contact angle $20^{\circ}$ ) and the hydrophobic $\left(88^{\circ}\right)$ substrate. No attempt was made to provide a complete set of measurements. In Fig. 4 the results are shown. On the horizontal axis the $\alpha$ hCG coverage is presented as a monolayer fraction. On the vertical axis the average activity per molecule-the immunorcactivity value divided by the corresponding $\alpha$ hCG coverage-is denoted in arbitrary units.

From this figure two trends are observed. First, the hydrophobicity of the surface determines the immunoreactivity of the adsorbed ahCG molecules. The immunoreactivity of a certain fraction of a monolayer of $\alpha$ hCG adsorbed at the hydrophobic surface is much larger than in the corresponding hydrophilic case. The second tendency visible from Fig. 4 is the decreasing immunoreactivity with increasing monolayer frac- $\uparrow$

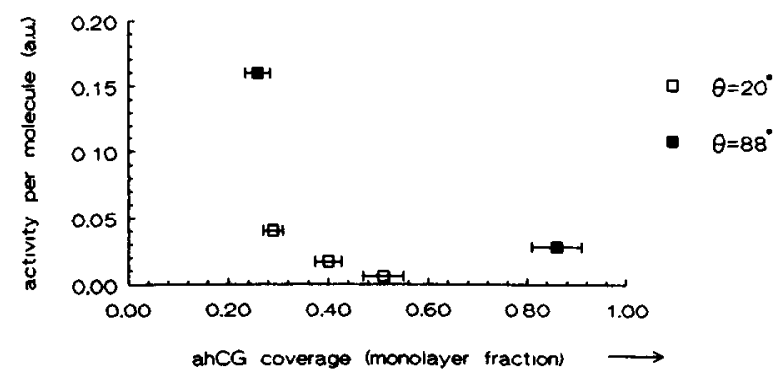

Fig. 4. ahCG activity per molecule as a function of substrate coverage, for hydrophilic (hollow marker) and hydrophobic (filled marker) substrates. Error bars not shown are covered by the marker.

tion of adsorbed $\alpha$ hCG. This effect was also reported in literature for an antibody coupled to a gel-like matrix (Matson, 1988). This tendency is observed for both the hydrophobic and the hydrophilic waveguide surface.

\section{Immunoreactivity with fixed ahCG density as a function of hydrophobicity}

\section{Immunoreaction saturation level as a function of} substrate hydrophobicity

In the previous section we found that the highest immunoreactivity could be obtained by adsorbing less than a monolayer of $\alpha \mathrm{hCG}$ on a hydrophobic waveguide surface. In this section $\cong \frac{1}{3}$ of the surface was covered with $\alpha$ hCG molecules (phase change $\cong 10 \cdot 2 \pi$ ), while the hydrophobicity of the waveguide surface was varied. The resulting immunoreaction saturation levels are shown in Fig. 5. Here, the phase changes corresponding to the saturation levels are normalized with respect to the phase change value caused by the $\alpha$ hCG adsorption. From this figure it is evident that increasing the hydrophobicity of the waveguide surface results in an increased immunoreactivity of the adsorbed $\alpha$ hCG layer. However, this increase is not linear, and only becomes significant in the contact angle region around $80^{\circ}$.

From Fig. 5 it can be seen that the maximal obtained immunoreaction at $\theta=88^{\circ}$ as compared to the $\alpha \mathrm{hCG}$ adsorption is $\approx 0 \cdot 16$, which is equivalent to $\approx 1 \cdot 5 \cdot 2 \pi$. In view of the molecular 


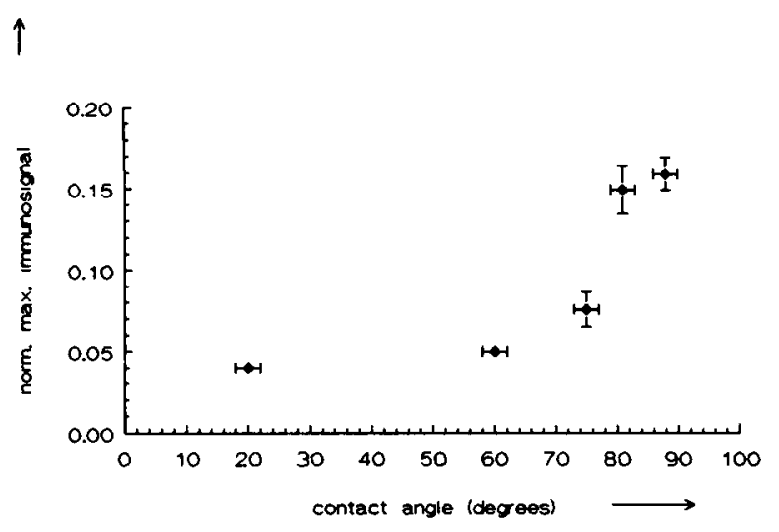

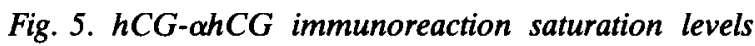
as a function of substrate contact angle. The ahCG coverage fraction is kept constant $(\approx 0.3$ monolayer $)$. The saturation levels are normalized with respect to the phase change value caused by the ahCG adsorption.

weight of hCG $(\approx 40 \mathrm{kD})$ and the two possible binding sites per $\alpha \mathrm{hCG}$ molecule, this value could be maximal $\approx 4 \cdot 5 \cdot 2 \pi$. Thus, in the best situation, only one third of the Fab's is capable of binding antigens.

\section{Immunoreaction affinity constant dependency upon substrate hydrophobicity}

A typical immunoreaction response as a function of hCG concentration is shown in Fig. 6a. The substrate contact angle value was $81^{\circ}$. The lowest detectable hCG concentration in this situation was $5 \cdot 10^{-11}$ Molar, corresponding to a phase change of $1 \cdot 10^{-2} \cdot 2 \pi$ in $\approx 20$ minutes. This corresponds to an average hCG layer growth of $1 \cdot 10^{-3} \mathrm{~nm}$ (Heideman et al., 1992 ${ }^{1}$. By normalizing the phase changes caused by the immunoreactions, a comparison between the hCG concentration dependent responses as a function of substrate hydrophobicity can be made. Here the equilibrium value of the immunoreaction saturation level is taken as 1 . In Figs. $6 \mathrm{~b}$, $\mathrm{c}$ and $\mathrm{d}$ some experimental results are shown, together with the theoretical curves given by the wellknown Langmuir description of the adsorption,

$[\mathrm{R}]+[\mathrm{A}] \stackrel{\boldsymbol{K}}{\rightleftarrows}[\mathrm{RA}]$, where $\Gamma=\mathrm{K} \cdot[\mathrm{A}] / 1+\mathrm{K} \cdot[\mathrm{A}]$

gives the fraction of the covered surface.

Here $\mathbf{R}$ and $A$ denote receptor and antigen molecule, respectively, and $\Gamma$ denotes the fraction of the active antibodies that has immunoreacted.
In these plots the value for the affinity constant $K$ was taken from the experimental data as judged from the point $\Gamma=\frac{1}{2}$. It is readily seen that the experimental results strongly deviate from the theoretical Langmuir curves.

In Fig. 7 the experimentally found immunoreaction affinity constants are plotted as a function of substrate contact angle. It appears that within the experimental error the affinity constant remains essentially stable over the range of contact angles.

\section{DISCUSSION}

From the results presented in the previous section (see Figs. 4 and 5) it is found that both the substrate hydrophobicity and the antibody density are of major importance for the immunoreactivity of the adsorbed antibody. A combination of a hydrophobic (contact angle $\geq 80^{\circ}$ ) substrate and only a fraction $(\approx 0.3)$ monolayer of antibodies gives the highest immunoreactivity. The decreasing immunoreactivity per molecule with increasing coverage fraction both on the hydrophobic and the hydrophilic substrate is probably due to the increasing crowding of Fab fragments, thereby limiting their binding capacity. The immunoreactivity of a certain fraction of a monolayer of $\alpha \mathrm{hCG}$ adsorbed at the hydrophobic substrate is much larger than that in the corresponding hydrophilic case (see Figs. 4 and 5). This means that a larger fraction of the Fab fragments that are present is active and is available for binding. Apparently, these Fab fragments are less hydrophobic than the Fc fragment, or the protein conformation on a hydrophobic substrate is more favourable for immunoreactions. However, a substrate-induced conformational change of the Fab fragments, resulting in loss of functionality, is very improbable in view of their high intrinsic stability (Huber, 1986). This is confirmed by the measurements presented in Fig. 7, showing that the experimentally found immunoreaction affinity constant remains essentially unchanged over the range of contact angles. We therefore believe that the increased immunoreactivity of adsorbed ahCG molecules with increasing waveguide hydrophobicity is mainly due to the circumstance that the orientation and not the conformation of the Fab fragments is dependent on the substrate hydrophobicity. 
(a)

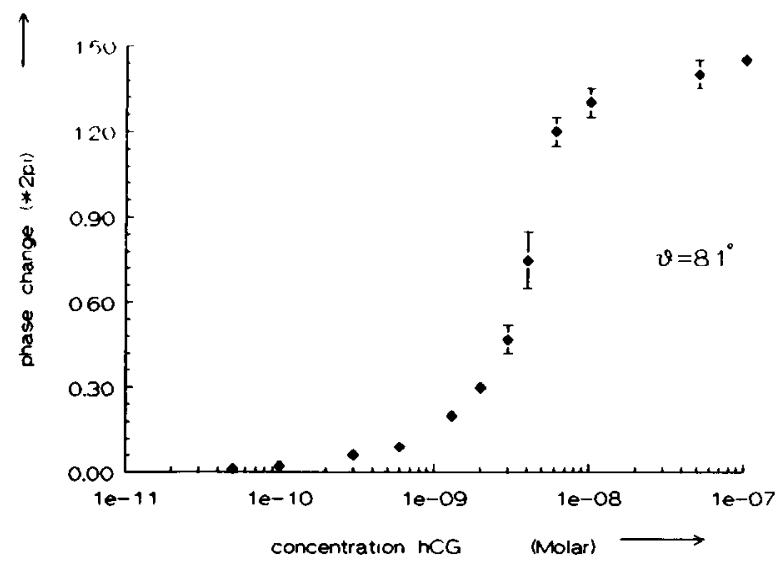

(b)

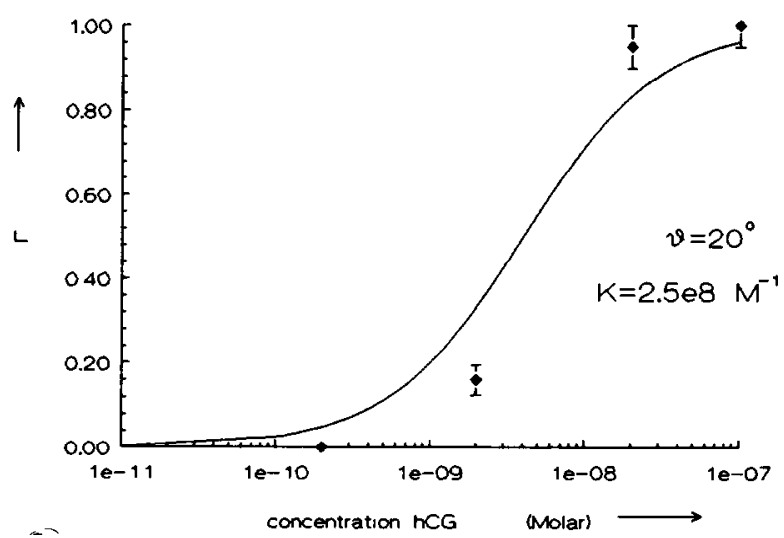

(c)

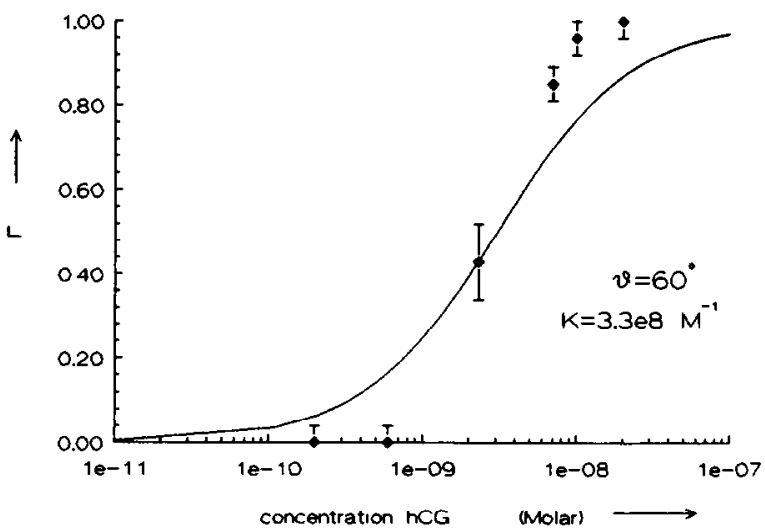

(d)

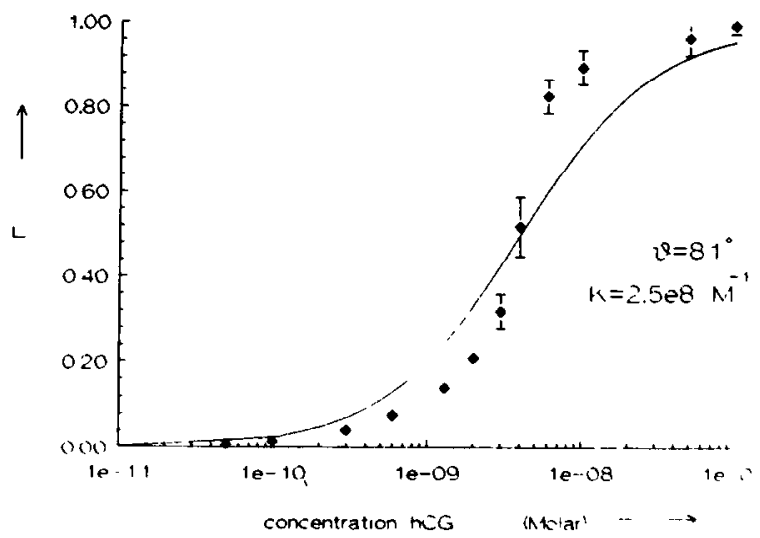

Fig. 6. The immunoreaction response as a function of hCG concentration, with the substrate hydrophobicity as parameter. (a) The equilibrium phase changes with contact angle value $\theta=81^{\circ}$. (b) through (d) The normalized immunoreaction responses together with the theoretical Langmuir curves for three different substrate contact angle values; $\theta=20^{\circ}, 60^{\circ}$ and $81^{\circ}$ respectively.

The monolayer adsorption measurements presented in Fig. 3 are in agreement with this suggestion. The signal obtained from the PIWI corresponding to the adsorption of a monolayer of proteins is quadratic in $n_{p}$ (Heideman, 1993); see equation (A3). From the literature it is known that the refractive index of large biomolecules such as antibodies is hardly dependent upon their molecular weight (Cuypers et al., 1983). In contrast, it is expected that the average refractive index of adsorbed proteins is dependent upon the protein conformation. This has indeed been demonstrated (Arwin, 1986). Therefore it is expected that a conformational change of the adsorbed antibodies strongly affects the magnitude of the signals obtained. However, the measured monolayer values for both ahCG and $\alpha$ hSA on a hydrophobic as well as on a hydrophilic substrate show a close similarity, as shown in Fig. 3. This confirms that no gross differences in conformation exist for the protein that is adsorbed to a hydrophilic or a hydrophobic substrate. An independent measurement of the refractive index of the adsorbed monolayers by methods such as proposed by Lukosz et al., (Lukosz et al., 1991) could verify this conjecture. The experimentally found immunoreaction affinity constants appear to be essentially stable over the range of contact angles used; see Fig. 7. Here these constants were taken from the normalized immunoreaction curves shown in Fig. 6, as judged from the point $\Gamma=\frac{1}{2}$. The experimental data themselves strongly deviate from the theoretical curves. Depletion of the solution can account for a deviation from the theoretical behaviour, as this is not included in the Langmuir theory. However, the deviations 


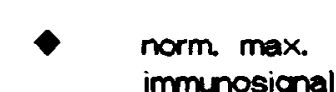

O K immunosignal

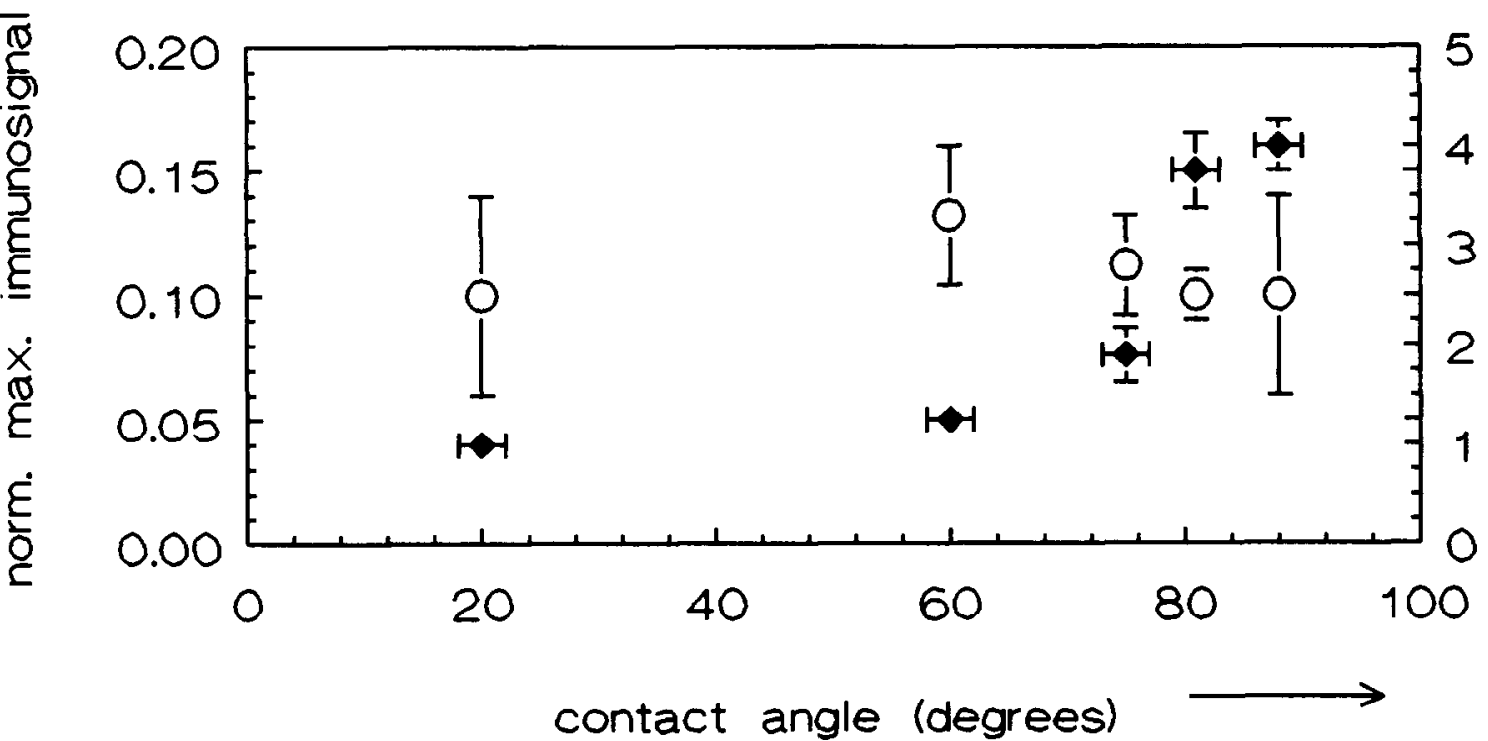

Fig. 7. The experimentally found immunoreaction affinity constants $K$ (hollow marker) and the normalized immunoreaction saturation levels (filled marker) as a function of substrate contact angle. For details see text.

do not significantly increase with lower hCG concentrations, so probably depletion does not completely account for the deviations. The shape of the curves strongly suggests positive cooperation in the binding process of the hCG molecules. Fitting the experimental data to an extended Langmuir formula including cooperativity (Scatchard et al., 1957) was possible, yielding cooperativity values of $\approx 4$. However, this value is very unrealistic in view of the maximal cooperativity value of 2 (meaning infinite cooperativity between the two binding sites). The real reason for the deviations therefore remains unclear. In order to test the possible effect of cooperativity it would be interesting to perform similar experiments with cleaved Fab-fragments.

\section{CONCLUSIONS}

The results presented in this paper illustrate the possibilities of the PIWI as a tool in studying biochemical surface reactions in order to obtain insight into the behaviour of macromolecules adsorbed at surfaces. Furthermore, this study demonstrates how the immunoreaction can be utilized as a tool in the investigation of protein adsorption phenomena. The experimental data contribute to a better knowledge of the adsorption behaviour of antibody molecules, which could be important in the coating of sensor surfaces. It was found that saturation of the adsorption process resulted in a monolayer of proteins for $\alpha \mathrm{hCG}, \alpha \mathrm{hSA}$ and hSA. The conformation of the monolayers does not seem to be dependent upon substrate hydrophobicity.

The substrate hydrophobicity and the antibody density were found to be of major importance for the immunoreactivity of the adsorbed antibodies. A combination of a hydrophobic (contact angle $\left.\geq 80^{\circ}\right)$ substrate and only a fraction $(\approx 0 \cdot 3)$ monolayer of antibodies gives the highest immunoreactivity. However, the immunoreaction affinity constant of the active antibodies is found to be essentially independent of the substrate hydrophobicity. From the experiments the picture emerges that the binding capacity of adsorbed antibodies is mainly determined by a combination of two factors, viz.:

(1) a proper orientation of antibodies such that Fab-fragments are optimally directed towards the solution, and 